\title{
Comment on: Modular titanium alloy neck failure in total hip replacement
}

\author{
SICOT J 2016, 2, 20, DOI: 10.1051/sicotj/2016009
}

\author{
Francesco Benazzo and Loris Perticarini* \\ Fondazione IRCCS Policlinico San Matteo, Pavia, Italy
}

Received 1 October 2016, Accepted 7 October 2016, Published online 17 March 2017

To the Editor of the SICOT-J Orthopaedic Journal,

I am writing to you as I would like to kindly express some considerations on the case-report of Dr. Marco Ceretti on "Modular titanium alloy neck failure in total hip replacement" published in your on-line journal last April (M. Ceretti and F. Falez SICOT J 2016, 2, 20).

The Authors report on " a case of a repetitive modular femoral neck prosthesis fracture"; they had initially revised a broken modular Metha stem with a MODULUS stem, neck $135^{\circ}$ long, head 36 medium in a 43 -year-old woman with body mass index (BMI) $38.6 \mathrm{~kg} / \mathrm{m}^{2}$ (weight $110 \mathrm{~kg}$, height $170 \mathrm{~cm}$ ); both stems presented modular necks in titanium alloy. The MODULUS stem was then revised with a Wagner monoblock uncemented titanium stem following the fracture at the neckstem junction.

This article reports a clinical case but it does not include any laboratory evidence in terms of fracture analysis to support the Authors' conclusions and to elucidate the mechanisms of failure. No retrieval analysis on the two stems seems to have been in fact carried out, such as standard macroscopic inspection and observations, standard corrosion testing, micro analysis of the implant alloy, light microscopy, Scanning Electron Microscopy (SEM) and patient's blood sample to check the release of metal ions.

Besides, several publications nowadays report comparative analysis of cobalt-chromium and titanium modular femoral necks, showing that the first material present "improved resistance against fretting" with "lower incidence of surface micromotions" (e.g. [1, 2]).

Nevertheless, the fracture of these two modular necks should be attributable to an error of indication and not to a failure of the devices themselves, meant to be used in patients with lower BMI.

\footnotetext{
*Corresponding author: Ioris.perticarini@gmail.com
}

The surgical technique of the MODULUS stem reports:

"Complications or failures of the total hip replacement may occur in heavy and highly active patients and high offset combinations. The surgeon should perform a careful evaluation of the patient's clinical condition and level of physical activity before performing hip replacement. Patients who are overweight and/or have high activity levels may not be candidates for hip replacement with modular stems. Alternative devices, such as monoblock hip stems, should be used, when possible, in these patients."

The available literature sources clearly support these indications on the use of modular stems in the current clinical practice:

"Among patients treated with the titanium alloy neck adapter, a combination of different parameters was identified as risk factors of implant failure. The parameters are intraoperative particle contamination of the cone connection, excessive loading due to a patient weight above $100 \mathrm{~kg}$ or high activity level, and male gender. In addition, the risk for failure rises with $\mathrm{CCD}$ angles of the cone adapter of $135^{\circ}$ and smaller."

"Contamination with cortical bone particles in the interface was found to be the main influence factor on the fatigue behaviour of the titanium alloy neck adapter." [1]

\footnotetext{
"Various circumstances such as large femoral head with a long varus neck, corrosion, patients' BMI, and activity level may participate in creating the necessary environment for fatigue failure of a modular hip replacement implant." [3]
} 
Furthermore, I would like to take this opportunity to kindly bring to the attention of your Editorial Office that no failures of the MODULUS stem are recorded in some series of publications concerning clinical experience with this medical device, as indicated in the following articles [4-7].

\section{Conflict of interest}

One of the authors is a consultant of Lima Corporate as a Scientific Advisor.

\section{References}

1. Grupp TM, Weik T, Bloemer W, Knaebel H-P (2010) Modular titanium alloy neck adapter failures in hip replacement - failure mode analysis and influence of implant material. Musculoskeletal Disorders 11, 3.

2. Jauch SY, Huber G, Haschke H, Sellenschloh K, Morlock MM (2014) Design parameters and the material coupling are decisive for the micromotion magnitude at the stem-neck interface of bi-modular hip implants. Med Eng Phys 36(3), 300-307.

3. Ollivier M, Parratte S, Galland A, Lunebourg A, Flecher X, Argenson J-N (2015) Titanium-titanium modular neck for primary THA. Result of a prospective series of 170 cemented THA with a minimum follow-up of 5 years. Orthop Traumatol Surg Res 101(2), 137-142.

4. Benazzo F, Cuzzocrea F, Stroppa S, Ravasi F, Dalla Pria P (2007) Modular stems in DDH. Hip Int 17(Suppl 5), S138-S141.

5. Benazzo F, Rossi SMP, Cecconi D, Piovani L, Ravasi F (2010) Mid-term results of an uncemented femoral stem with modular neck options. Hip Int 20(4), 427-433.

6. Dagnino A, Grappiolo G, Benazzo FM, Learmonth ID, Portinaro N, Spotorno L (2012) Medium-term outcome in patients treated with total hip arthroplasty using a modular femoral stem. Hip Int May-Jun 22(3), 274-279.

7. Benazzo FM, Piovani L, Combi A, Perticarini L (2015) MODULUS stem for developmental hip dysplasia: long-term follow-up. J Arthroplasty 30(10), 1747-1751.

Cite this article as: Benazzo F \& Perticarini L (2017) Comment on: Modular titanium alloy neck failure in total hip replacement. SICOT J, 3,23 